\title{
Análisis Manual Aproximado y Exacto de Pórticos Espaciales mediante Cargas Descompuestas
}

\author{
Agustín G. Lacort \\ Departamento de Arquitectura. ETSA, Universidad del País Vasco (UPV-EHU), \\ Plaza de Oñati, 2, 20018 San Sebastián, Donostia-España
}

Recibido Abr. 01, 2010; Aceptado Jun. 01, 2010; Versión Final recibida Dic. 30, 2010

\begin{abstract}
Resumen
El presente estudio describe dos procedimientos iterativos de análisis estructural en régimen lineal y teoría de primer orden que permiten estudiar de manera aproximada y exacta determinados emparrillados planos y espaciales con varios desplazamientos independientes sin tener que resolver sistemas de ecuaciones. Con el procedimiento aproximado se consiguen unos resultados suficientemente precisos realizando pocas iteraciones, siendo el error cometido estimable en todo momento. En el procedimiento exacto se utilizan dos tablas de aplicación simple, práctica y fácil de ser implantadas manualmente en una aplicación informática. Con dichas tablas se obtienen las deformadas realizando un número de operaciones menor que el requerido por el álgebra matricial.
\end{abstract}

Palabras clave: analisis estructural, métodos iterativos, pórticos espaciales, métodos de equilibrio

\section{Approximate and Exact Manual Analysis of Space frames by Decomposition of Loads}

\begin{abstract}
This study describes two iterative structural analysis procedures under a linear system within a first-order theory that permits the approximate and exact study of flat frames and space frames with independent displacements without having to solve any system of equations. The approximate system provides sufficiently accurate results in few iterations, and the error can be estimated at any time. The exact procedure uses two simple, practical application tables that can be manually implemented in software. The tables provide deformed meshes with fewer operations than those required by matrix algebra.
\end{abstract}

Keywords: structural analysis, iterative methods, three-dimensional frames, equilibrium methods 


\section{INTRODUCCIÓN}

El análisis estático lineal y en teoría de primer orden de pórticos continuos ordinarios de edificación realizado mediante programas informáticos permite conocer rápidamente y con total precisión los resultados de los grandes sistemas de ecuaciones lineales derivados de los planteamientos del cálculo. La eficacia de estas aplicaciones informáticas ha alejado al ingeniero proyectista de los métodos manuales simplificados, métodos que tradicionalmente han servido para desarrollar el sentido estático y el de los órdenes de magnitud de los esfuerzos producidos. Ambos aspectos son esenciales en la formación de un estructuralista, ya que como señala Fernández Casado, "la intuición y la experiencia son las dos fuentes vivas a las que ha de acudir el que proyecta" (Fraile de Lerma et al, 2008). En la actualidad, existe un interés creciente en desarrollar métodos manuales simplificados derivados del comportamiento estructural según la teoría de primer orden, que sirvan para predimensionar modelos formados con pocos elementos y también para comprobar esfuerzos locales de pórticos analizados previamente con programas informáticos. Estos métodos deben ser sencillos de aplicar y semiautomáticos en sus operaciones con objeto de reducir la probabilidad de cometer errores de cálculo. Además, los resultados que obtienen deben estar del lado de la seguridad y deben ser una referencia cualitativa de los resultados exactos, ya que como comenta Portero et al (2004) "sin ese primer control de la situación (el uso de fórmulas simplificadoras), los métodos sofisticados serían peligrosos". Existen numerosos procedimientos, muchos de ellos concebidos en épocas pasadas, aunque según Grinter (1969), ninguno de ellos por sí solo es conveniente para analizar todas las situaciones posibles. La mayor dificultad que tienen es determinar con suficiente precisión los esfuerzos derivados de los desplazamientos, caso de existir, lo que implica resolver el sistema de ecuaciones de equilibrio de planta. Los métodos del portal y del voladizo calculan estos esfuerzos considerando exclusivamente las exigencias estáticas del modelo y suponiendo conocida la localización de los puntos de inflexión. Calavera (1999) sugiere aproximar los resultados obtenidos con estos métodos a los valores exactos utilizando unas tablas propuestas por Bull y Svud. Otros métodos aproximados de equilibrio, como son el de Kani, el de Takabeya o el de las aproximaciones sucesivas, resuelven el sistema de ecuaciones de Gehler realizando múltiples iteraciones. Sin embargo, estas y otras estrategias para simplificar el modelo que se analiza no están concebidas para estudiar figuras espaciales debido a la complejidad de cálculo que ello implica.

Otras líneas de investigación actuales y más relevantes persiguen conocer con mayor detalle el comportamiento estructural de los pórticos. Esto supone contemplar en el análisis la naturaleza semirrígida de las uniones y considerar diferentes respuestas estructurales, como son la dinámica no lineal, la plástica, el comportamiento en teoría de $2^{\circ}$ orden, frente al fuego, etc. Dada la enorme dificultad que conlleva estudiar estos fenómenos existe un interés en desarrollar también métodos simplificados que aporten soluciones razonablemente exactas a estas cuestiones pero empleando un número reducido de operaciones. En algunos trabajos recientes como el de Giordano et al. (2007) o el de Zubydan (2010) se proponen expresiones y modelos de cálculo simplificados. En otros, como en el de Aristizabal-Ochoa (2009) y en el de Günay y Sucuoglu (2010), se realizan análisis lineales en teoría de primer orden de modelos formados con elementos cuyo comportamiento contemple la respuesta estructural comentada anteriormente, es decir, aquella que no se detecta en un análisis de primer orden convencional.

En este trabajo se exponen dos procedimientos iterativos, uno aproximado y otro exacto que complementan estas líneas de investigación ya que sirven para analizar pórticos planos y tridimensionales en teoría de primer orden sin resolver sistemas de ecuaciones. Ambos métodos son de equilibrio y necesitan un número de operaciones menor que el requerido por el álgebra matricial. El trabajo proviene de otro anterior expuesto en el último Congreso de ACHE en Valencia (España), válido para determinados emparrillados espaciales. Su planteamiento era parecido al del método de las aproximaciones sucesivas, pero enfocado desde un punto de vista diferente. Las acciones exteriores se descomponían en un conjunto de estados de carga, que se denominaron "estados primarios" (Fig.1a) de manera que produjeran solicitaciones y deformadas, inmediatas de calcular manualmente. Cada estado primario estaba formado por una acción "activa" y por varias "restrictivas". Mientras la acción activa, $\mathrm{m}_{n}^{\mathrm{a}} \circ \mathrm{f}_{\mathrm{n}}^{\mathrm{a}}$ "provocaba" 
el giro de algún nudo $\mathrm{n}$ o bien un desplazamiento independiente, las restrictivas, representadas en las figuras en línea discontinua, evitaban el resto de los movimientos nodales. Para conocer estos estados primarios de manera aproximada y sin resolver sistemas de ecuaciones se sumaban múltiples estados de carga primarios elegidos eficazmente. Las acciones activas de los estados primarios que iniciaban el sumatorio coincidían con las acciones exteriores originales (Fig.1b). La diferencia entre la suma de estos estados y las acciones originales eran las acciones restrictivas $r_{i}$. En una primera iteración, las acciones $r_{i}$ se eliminaban añadiendo al conjunto otros estados cuyas acciones activas valieran - $r_{i}$ (Fig.1c). Sumando los estados primarios utilizados hasta el momento se obtenía un estado de carga más parecido al original que antes de iterar, ya que los valores de las acciones restrictivas eran menores. Tras sucesivas iteraciones, las acciones restrictivas tendían a desaparecer y la suma de las acciones activas de todos los estados implicados en el sumatorio se aproximaba a las acciones activas del estado de carga original.

a)

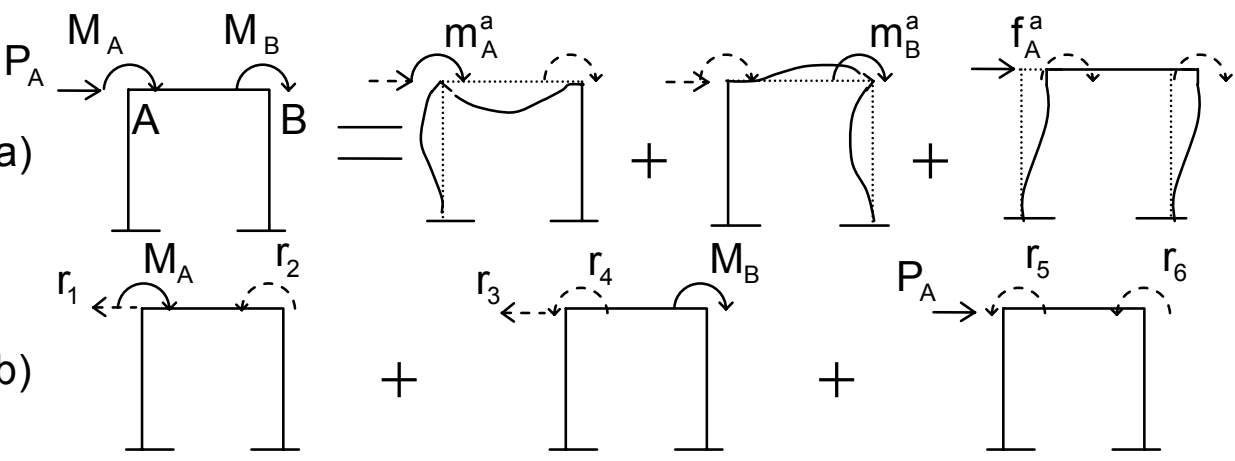

c)

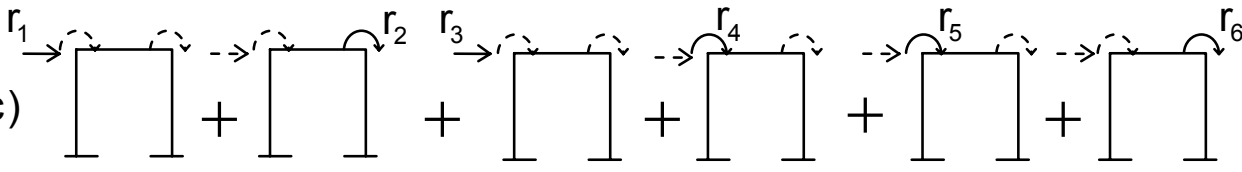

Fig. 1. Planteamiento iterativo propuesto: a) Descomposición de las cargas en estados primarios;

b) Estados primarios iniciales; c) Estados primarios de la primera iteración

A raíz de los ejemplos que se analizaron, se observó que el número de iteraciones necesario para conseguir unos resultados aceptables dependía del grado de desplazabilidad de la figura. Cuando la estructura era indesplazable bastaba con realizar tres iteraciones, mientras que cuando existía un desplazamiento independiente, eran necesarias siete. No se obtuvo ninguna conclusión en el resto de los casos. El presente estudio propone una modificación que permite analizar estructuras con múltiples desplazamientos siempre que estos se encuentren contenidos en el mismo plano. La modificación consiste en eliminar las acciones restrictivas de dos maneras diferentes, lo que da lugar a los dos procedimientos iterativos comentados anteriormente. Ambos métodos están gobernados por unas reglas nemotécnicas relativamente poco sofisticadas, por lo que resultan adecuados para ser implantados por el usuario en una aplicación informática. En ambos casos se comienza con unos estados primarios iniciales elegidos como en el trabajo anterior. En una primera etapa se neutraliza parte de las acciones restrictivas y en una segunda se eliminan las restantes. Los procedimientos se explican cualitativamente a continuación aplicándolos a un pórtico plano de dos desplazamientos. Finalmente, a modo de ejemplo, se obtienen las acciones activas de un pórtico espacial utilizando unas reglas nemotécnicas.

\section{METODOLOGÍA APLICADA}

\section{Explicación cualitativa}

Sea el pórtico de la figura $2 a$ sometido a un conjunto $Q$ de cargas cuyas acciones activas se desea conocer. Estas acciones se obtienen sumando las activas de un conjunto de estados primarios. El sumatorio comienza con dos estados de carga $Q_{M}$ y $Q_{P}$. Las acciones activas de 
estos dos estados son los momentos $M_{n}$ y las fuerzas $P_{n}$ de $Q$, respectivamente. Las acciones restrictivas existentes en $Q_{M}$ y $Q_{P}$ constituyen la diferencia entre la suma de estos dos conjuntos y $Q$. Dichas acciones se eliminan en dos etapas que se describen a continuación. En la etapa 1 se eliminan los momentos restrictivos de $Q_{M}$ (Fig. 2b). Para ello se añaden al conjunto diferentes estados primarios cuyas acciones activas son momentos de igual valor pero de signo contrario a los restrictivos existentes. Se designa con $\mathrm{m}_{\mathrm{nM}}^{\mathrm{a}}$ a la suma de los momentos activos resultantes en cada nudo $\mathrm{n}$ obtenidos en esta etapa (Fig.2c). La adición de los estados primarios hace que se modifiquen las fuerzas restrictivas de $Q_{M}$ en $B$ y $A$, alcanzando las magnitudes $B M$ y $A M$. En la etapa 2 se elimina el resto de las acciones restrictivas de la estructura. Añadiendo dos estados primarios cuyos valores son BM y AM veces los estados I y II respectivamente (Figs.2e y $2 \mathrm{~g}$ ), se suprimen las fuerzas BM y AM, quedando por eliminar las acciones restrictivas de los estados primarios de la figura $2 \mathrm{~d}$. Esto se realiza calculando previamente las acciones activas de los estados I y II. El cálculo comienza haciendo desaparecer los momentos $r_{n m}$, lo que da lugar a los momentos activos parciales $m_{n B}^{a}$ y $m_{n A}^{a}$ y a las fuerzas restrictivas $B B, A B$ y $B A, A A$ (Figs. $2 f$ y $2 h$ ). Todas estas fuerzas son menores que la unidad $y$ valen las restrictivas $s_{n m}$ más las producidas al eliminar $r_{n m}$. A continuación se suprimen dichas fuerzas añadiendo estados primarios similares a I y $\mathrm{II}$, de manera que sus fuerzas activas coincidan con las restrictivas a anular pero cambiadas de signo. Procediendo repetidamente de esta manera, se reducen progresivamente las fuerzas restrictivas $y$, a la vez, las fuerzas activas acumuladas se parecen cada vez más a las de las figuras 2 e y $2 \mathrm{~g}$. Al multiplicarlas por $F_{A}$ y $F_{B}$, dan lugar a las fuerzas activas $f_{A}^{a}$ y $f_{B}^{a}$ asociadas al estado de cargas $Q$. Los momentos activos totales $m_{n}^{a}$ (Fig $2 i$ ) se obtienen multiplicando $f_{A}^{a}$ y $f_{B}^{a}$ por los momentos $m_{n A}^{a}$ y $m_{n B}^{a}$ respectivos y sumando estos resultados a $m_{n M}^{a}$. Por tanto, es necesario determinar los momentos activos parciales $m_{n M}^{a}, m_{n A}^{a} y$ $m_{n B}^{a}$ y las fuerzas activas $f_{A}^{a}$ y $f_{B}^{a}$, valores que se propone determinar de manera aproximada y de manera exacta.

El procedimiento aproximado calcula los momentos parciales y las fuerzas activas mediante sucesivas iteraciones realizadas con matrices diferentes. En general existe una matriz por cada plano definido por los ejes de referencia globales de la estructura. Estas matrices se calculan a partir de la forma general del pórtico y de unas reglas nemotécnicas que se explicarán en el ejemplo de cálculo. Cada matriz relaciona los momentos activos con los restrictivos existentes entre dos iteraciones consecutivas. La precisión de los resultados obtenidos es similar a la que se consigue con el método de Cross, ya que las operaciones numéricas son las mismas. A partir de la tercera iteración los momentos restrictivos que faltan por eliminar se pueden despreciar. Por otra parte, la matriz que se propone para calcular las fuerzas activas se obtiene a partir de los valores de los momentos activos determinados anteriormente. Homólogamente al caso anterior, dicha matriz relaciona las fuerzas restrictivas existentes con las activas que se añaden en cada iteración, y el número de iteraciones que hay que realizar para eliminar razonablemente todas las fuerzas restrictivas es mayor que tres. Parece razonable emplear este método para analizar manualmente ciertos pórticos con pocos elementos ya que el número de operaciones a realizar puede ser asumible por el usuario.

El procedimiento exacto proporciona los valores estrictamente necesarios de los momentos parciales activos y de las fuerzas activas para anular completamente todas las acciones restrictivas. Estas acciones las elimina en sucesivos pasos y de manera diferente al planteamiento anterior, "arrinconando" progresivamente las acciones residuales en la estructura hasta hacerlas desaparecer. A continuación se describe de manera cualitativa este procedimiento aplicado a la supresión de las fuerzas $B B$ y $A B$ de la figura $2 f$. Se comienza eliminando la fuerza $A B$, añadiendo a la figura $2 f$ un conjunto de acciones formado por acciones activas y por una fuerza restrictiva en $B$, que se obtienen modificando adecuadamente las acciones de la figura $2 \mathrm{~h}$. Para eliminar en dicha figura la fuerza AA, se añade $A A$ veces las acciones de la figura $2 \mathrm{~g}$. Eliminando los momentos restrictivos añadidos como se hizo anteriormente en la figura $2 \mathrm{~h}$, aparece una nueva fuerza restrictiva en $A$ de valor $A A^{2}$. 


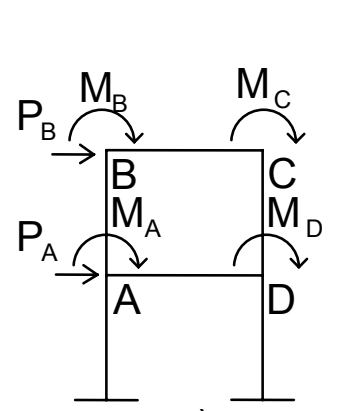

a)

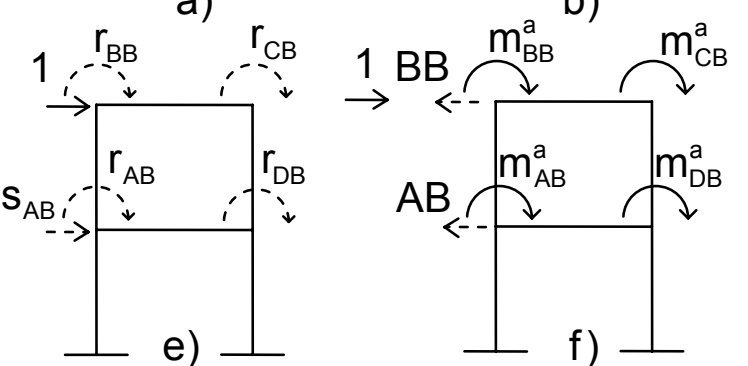

e)

$$
\mathrm{m}_{\mathrm{BM}}^{\mathrm{a}}+\mathrm{f}_{\mathrm{B}}^{\mathrm{a}} \mathrm{m}_{\mathrm{BB}}^{\mathrm{a}}+\mathrm{f}_{\mathrm{A}}^{\mathrm{a}} \mathrm{m}_{\mathrm{BA}}^{\mathrm{a}}=\mathrm{m}_{\mathrm{B}}^{\mathrm{a}}
$$

$\mathrm{M}_{\mathrm{C}}$$$
m_{A M}^{a}+f_{B}^{a} m_{A B}^{a}+f_{A}^{a} m_{A A}^{a}=m_{A}^{a} \quad m_{D}^{a}=m_{D M}^{a}+f_{B}^{a} m_{D B}^{a}+f_{A}^{a} m_{D A}^{a}
$$

c)
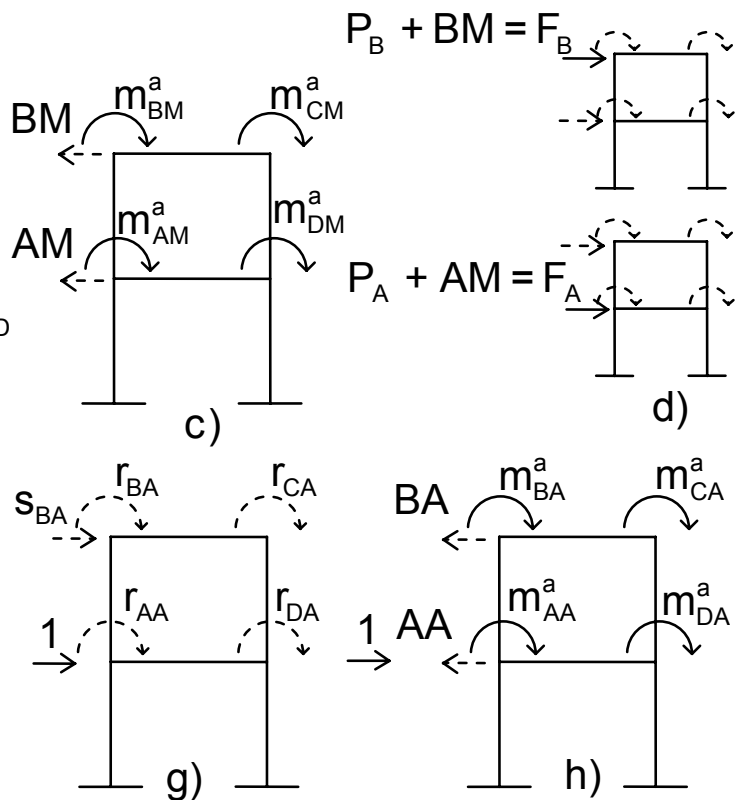

h)

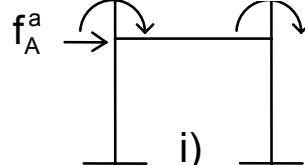

Fig. 2. Acciones activas de un pórtico desplazable: a): acciones exteriores $Q$; b) estados primarios de $Q_{M}, c$ ) acciones activas y restrictivas de la etapa 1, d): estados primarios iniciales de la etapa 2. e),g): estados primarios I y II; f),h): estados I y II transformados; i) acciones activas totales.

Suprimiendo $\mathrm{AA}^{2}$ mediante el mismo procedimiento, se obtienen nuevos momentos activos y nuevas fuerzas restrictivas en $A$ y $B$, que son $A A$ veces la anterior. Continuando este proceso indefinidamente, se acumula en $A$ un conjunto de fuerzas activas que forman una serie geométrica cuya suma total de términos (1) representa el valor exacto de la fuerza activa en A. En la figura 3a se muestran las fuerzas resultantes derivadas de este proceso al eliminar AA de la figura $2 \mathrm{~h}$, y en la $3 b$ se muestran las fuerzas que se obtienen en la figura $2 f$ al eliminar $A B$. En la figura $3 b$, la fuerza restrictiva de $B$ se elimina mediante el mismo procedimiento, obteniéndose finalmente las fuerzas activas totales que aparecen en 3c. Si inicialmente hubiera existido una fuerza $F_{A}$ en $A$, dicha fuerza debería haberse intercalado tal y como aparece en $3 \mathrm{~d}$.

$$
1+A A+A A^{2}+A A^{3}+\ldots \ldots \ldots \ldots=\frac{1}{1-A A}=A A_{1}
$$

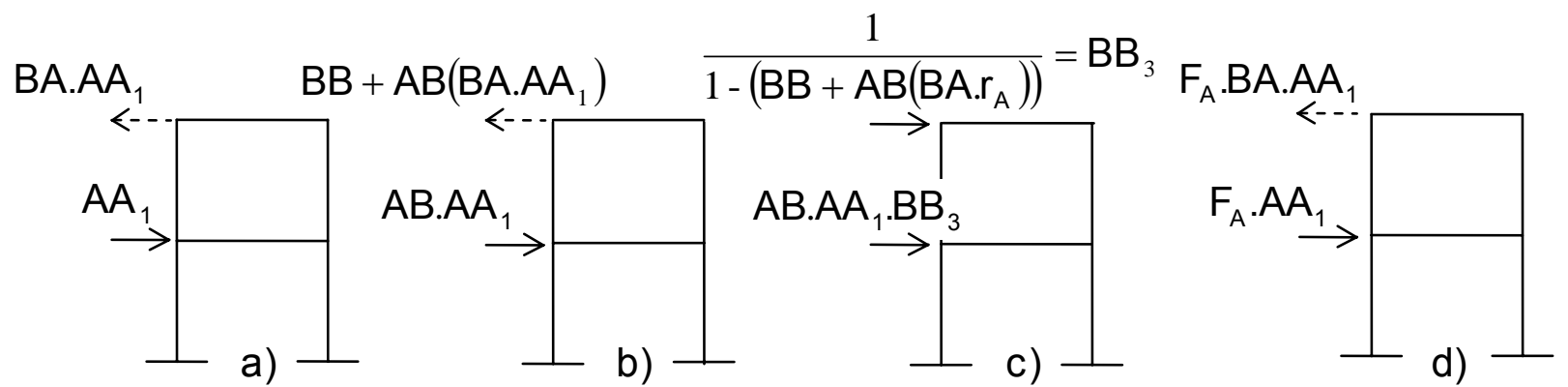

Fig. 3. Cálculo exacto de acciones activas del estado I. Valores obtenidos durante el proceso 


\section{Tablas de aplicación}

Cuando la estructura presenta más de dos desplazamientos independientes, la dificultad para explicar el procedimiento exacto crece enormemente. Por esta razón se propone utilizar dos tablas de aplicación conjunta que sirvan para determinar de forma sistemática las fuerzas activas $f_{n}^{a}$. Las tablas pueden emplearse para analizar indistintamente figuras planas y espaciales. El número de desplazamientos independientes de la estructura condiciona la extensión de estas tablas, cuyas casillas se rellenan según unas determinadas reglas nemotécnicas. La primera de las dos tablas tiene forma escalonada y consta de $2 n$ columnas y $3 n$ filas, siendo $n$ el máximo número de desplazamientos independientes que puede tener el pórtico. A modo de ejemplo se muestra la Tabla 1, en la que los nudos que condicionan los desplazamientos están numerados de abajo hacia arriba con las letras A, B y C. A la izquierda de la columna $a_{1}$ se han representado los estados primarios unitarios I, II y III que son homólogos a los de las figuras $2 \mathrm{e} \mathrm{y} 2 \mathrm{~g}$, en los que el vector indica la posición de la fuerza activa unitaria. En la columna $a_{1}$ se disponen las fuerzas restrictivas que aparecen después de eliminar los momentos restrictivos $r_{n A}, r_{n B}$ y $r_{n C}$, que son homólogas a las de las figuras $2 \mathrm{f}$ y $2 \mathrm{~h}$. Mediante unas operaciones que se describen a continuación, las fuerzas de la columna $a_{1}$ se transforman en otras representadas con asteriscos.

Tabla 1. Cálculo de fuerzas activas y restrictivas parciales en pórticos desplazables

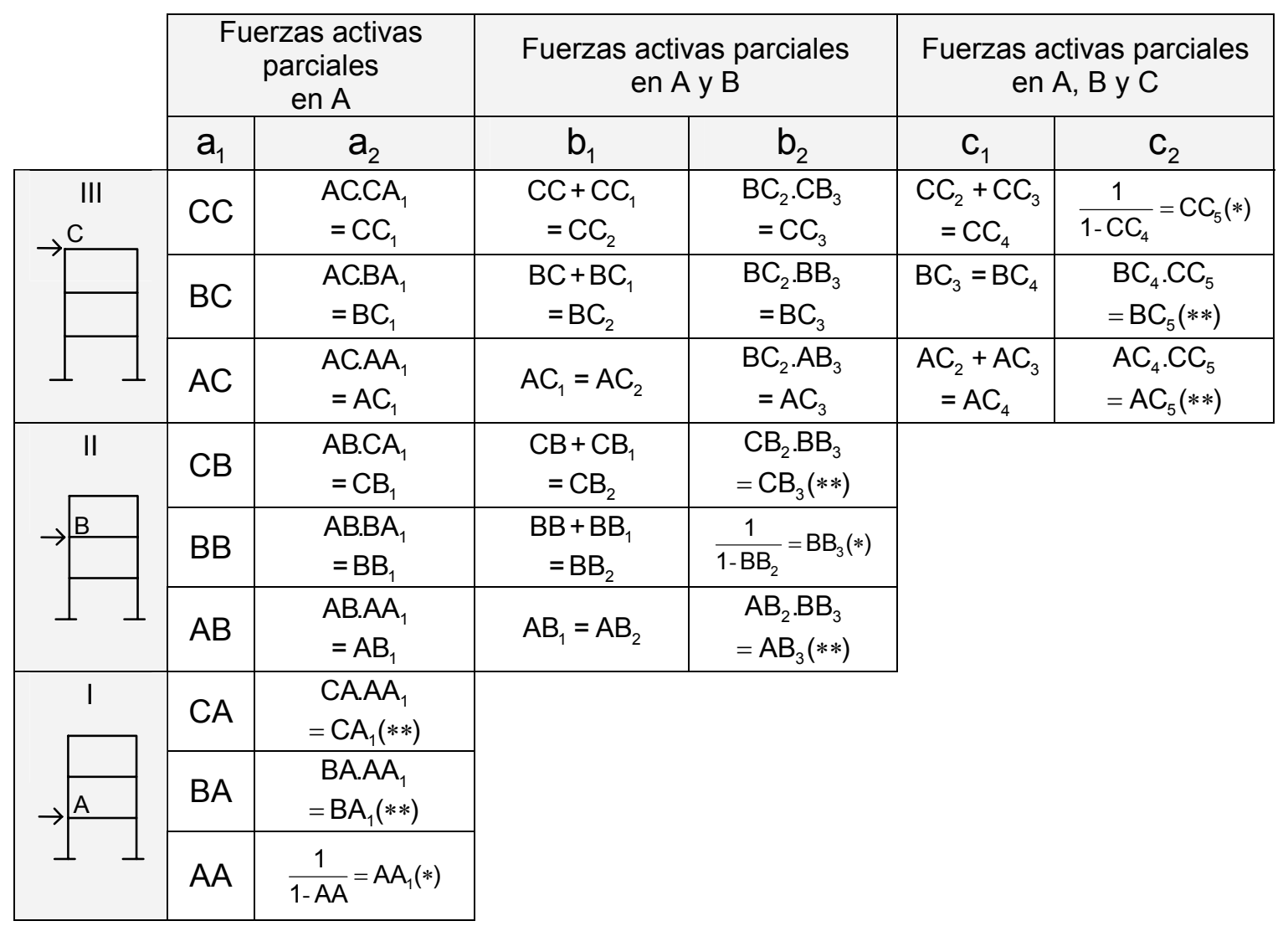

Partiendo de los datos de $a_{1}$ se obtienen los de las casillas que forman la columna $a_{2}$. Primero se calcula la casilla marcada con $(*)$, después las que tienen $(* *)$ y en función de ellas, las demás. Con los datos de las columnas $a_{1}$ y $a_{2}$, en la columna $b_{1}$ se obtienen las fuerzas activas en $A$ y las restrictivas acumuladas en $B$ y $C$ de los casos II y III. Con estos datos, en la columna $b_{2}$ se calculan las fuerzas activas y restrictivas que resultan de eliminar la restrictiva en el nudo $B$ (casos II y III). Como en $\mathrm{a}_{2}$, primero se calculan las casillas con asteriscos y en función de ellas, las demás. Con los datos de $b_{1}$ y $b_{2}$ se obtienen en $c_{1}$ las fuerzas activas en $A$ y $B$ y la restrictiva en C del estado III. 
Tabla 2. Cálculo de fuerzas activas totales en pórticos desplazables

\begin{tabular}{|c|c|c|c|c|c|c|c|c|}
\hline$a_{1}$ & $a_{2}$ & $a_{3}$ & $b_{1}$ & $b_{2}$ & $b_{3}$ & $\mathrm{C}_{1}$ & $\mathrm{C}_{2}$ & $\mathrm{C}_{3}$ \\
\hline- & $\mathrm{CA}_{1}$ & $\mathrm{~F}_{\mathrm{A}} \cdot \mathrm{CA}_{1}$ & - & $\mathrm{CB}_{3}$ & $\left(\mathrm{~F}_{\mathrm{A}} \cdot \mathrm{BA}_{1}+\mathrm{F}_{\mathrm{B}}\right) \cdot \mathrm{CB}_{3}$ & $F_{\mathrm{c}}$ & $\mathrm{CC}_{5}$ & $\begin{array}{c}\left(F_{A} \cdot C A_{1}+\left(F_{A} \cdot B A_{1}+F_{B}\right) \cdot C B_{3}+F_{C}\right) \cdot C C_{5} \\
=s_{6}\end{array}$ \\
\hline- & $\mathrm{BA}_{1}$ & $\mathrm{~F}_{\mathrm{A}} \cdot \mathrm{BA}_{1}$ & $\mathrm{~F}_{\mathrm{B}}$ & $\mathrm{BB}_{3}$ & $\begin{array}{c}\left(\mathrm{F}_{\mathrm{A}} \cdot \mathrm{BA}_{1}+\mathrm{F}_{\mathrm{B}}\right) \cdot \mathrm{BB}_{3} \\
=\mathrm{s}_{4}\end{array}$ & - & $\mathrm{BC}_{5}$ & $\begin{array}{c}\left(\mathrm{F}_{\mathrm{A}} \cdot \mathrm{CA}_{1}+\left(\mathrm{F}_{\mathrm{A}} \cdot \mathrm{BA}_{1}+\mathrm{F}_{\mathrm{B}}\right) \cdot \mathrm{CB}_{3}+\mathrm{F}_{\mathrm{C}}\right) \cdot \mathrm{BC}_{5} \\
=\mathrm{s}_{5}\end{array}$ \\
\hline $\mathrm{F}_{\mathrm{A}}$ & $\mathrm{AA}_{1}$ & $\begin{array}{l}\mathrm{F}_{\mathrm{A}} \cdot \mathrm{AA}_{1} \\
=\mathrm{s}_{1}\end{array}$ & - & $\mathrm{AB}_{3}$ & $\begin{array}{c}\left(\mathrm{F}_{\mathrm{A}} \cdot \mathrm{BA} \mathrm{A}_{1}+\mathrm{F}_{\mathrm{B}}\right) \cdot \mathrm{AB}_{3} \\
=\mathrm{s}_{2}\end{array}$ & - & $\mathrm{AC}_{5}$ & $\begin{array}{c}\left(F_{A} \cdot C A_{1}+\left(F_{A} \cdot B A_{1}+F_{B}\right) \cdot C B_{3}+F_{C}\right) \cdot A C_{5} \\
=s_{3}\end{array}$ \\
\hline
\end{tabular}

Finalmente, en $\mathrm{C}_{2}$ se obtienen las fuerzas activas para el caso III. A partir de estos resultados, en la Tabla 2 se calculan las fuerzas activas totales $f_{n}^{a}$. Dicha tabla se utiliza para introducir en el procedimiento las fuerzas $F_{n}$ que son homólogas a las de la figura $2 d$. Se comienza situando en las casillas de las columnas $a_{1}, b_{1}$ y $c_{1}$ las fuerzas $F_{n} y$, en las columnas $a_{2}, b_{2}$ y $c_{2}$, los valores de las casillas con asterisco calculadas en la Tabla 1 . Con los valores de $a_{1}$ y $a_{2}$ se calcula en la columna $a_{3}$ una fuerza activa parcial en A y otras restrictivas parciales en $B$ y $C$, todas ellas debidas a $F_{A}$. Las casillas de las columnas $n_{3}$ restantes se calculan añadiendo a cada fuerza $F_{n}$ la suma de las casillas $(n-1)_{3}$ situadas a la altura de dicha fuerza y a su izquierda, y multiplicando este resultado por las casillas de la columna $n_{2}$ correspondiente. Finalmente las fuerzas activas valen (2), quedando expresadas en función de las fuerzas $F_{n}$.

$$
\begin{aligned}
& \mathrm{f}_{\mathrm{C}}^{\mathrm{a}}=\mathrm{s}_{6} \\
& \mathrm{f}_{\mathrm{B}}^{\mathrm{a}}=\mathrm{s}_{4}+\mathrm{s}_{5} \\
& \mathrm{f}_{\mathrm{A}}^{\mathrm{a}}=\mathrm{s}_{1}+\mathrm{s}_{2}+\mathrm{s}_{3}
\end{aligned}
$$

Esta aplicación tabular también puede utilizarse para determinar los valores exactos de los momentos activos parciales. En la columna $a_{1}$ de una Tabla 1 adecuada para emplearla en el pórtico del ejemplo, habría que disponer los momentos restrictivos con signo contrario de los estados de carga de la figura $2 b$, suponiendo que $M_{n}$ fueran momentos activos unitarios. En la tabla 3 se muestran los datos de las casillas inferiores que habría que disponer en dicha tabla. $\theta_{\mathrm{A}}^{\prime}$ es el giro de A producido por el estado primario cuya acción activa es un momento unitario en

\begin{tabular}{|c|c|c|c|}
\hline & & \\
\hline & & $a_{1}$ & $a_{2}$ \\
\hline \multirow{2}{*}{\multicolumn{2}{|c|}{$\left.\right|^{\mathrm{B}} \mathrm{B}^{\mathrm{B} \uparrow} \prod^{\mathrm{C}}$}} & $D A=-\theta_{A}^{\prime} \cdot \frac{2 E I}{L}$ & DA. $1=\mathrm{DA}_{1}$ \\
\hline & & $\mathrm{CA}=0$ & $\mathrm{CA} \cdot 1=0=\mathrm{CA}_{1}$ \\
\hline \multirow[t]{2}{*}{$\mathrm{A}$} & $\overrightarrow{\mathrm{DA}^{\prime}}{ }^{\mathrm{D}}$ & $B A=-\theta_{A}^{\prime} \cdot \frac{2 E I}{L}$ & BA. $1=$ BA $_{1}$ \\
\hline & & $A A=0$ & $\frac{1}{1-\mathrm{AA}}=1=\mathrm{AA}_{\mathrm{A}}$ \\
\hline
\end{tabular}
A.

Tabla 3. Momentos activos y restrictivos producidos por el giro de un nudo A

El procedimiento es suficientemente sencillo y sistemático para poderlo implementar en una aplicación informática por el propio usuario, pudiendo utilizarse igualmente cuando en el análisis 
se consideren los acortamientos y alargamientos de las barras. Por otra parte, se ha observado que a medida que aumenta el número de nudos, el método aproximado resulta más práctico que el exacto ya que el número de operaciones que hay que realizar con este último crece enormemente. También se ha observado que el método exacto puede ser más útil que el aproximado cuando se analiza el comportamiento de la estructura frente a diferentes estados de carga, ya que con dicho método se obtiene la expresión de las acciones activas en función de las cargas actuantes.

\section{RESULTADOS Y DISCUSIÓN}

\section{Cálculo de un pórtico espacial}

Utilizando los procedimientos expuestos anteriormente se van a calcular las acciones activas de la estructura espacial de la figura 4a que tiene dos desplazamientos y que está formada por barras biempotradas de $4 \mathrm{~m}$. de longitud y de secciones cuadradas. Las matrices iterativas y las acciones restrictivas de los estados I y II se determinan aplicando las reglas nemotécnicas que aparecen en las figuras 4 y 5 . De este modo se pretende relacionar cualitativamente las operaciones realizadas con un esquema de la estructura, como sucede en los métodos de Cross y de Takabeya. En la figura $4 \mathrm{~b}$ se ha representado en el centro de cada barra girable un conjunto $\alpha$ de casillas con forma de "T" cuya orientación varía con la de la barra. Los valores de las casillas a y $c$ de $a$ (Fig.4d) dependen de la longitud $L$ del tramo. Los valores de las casillas b, $\mathrm{d}$ y e de todas las barras representan las rigideces a flexión y torsión respecto de los ejes utilizados (Fig.4c). Los resultados obtenidos en el cálculo se han simplificado a partir del segundo decimal.

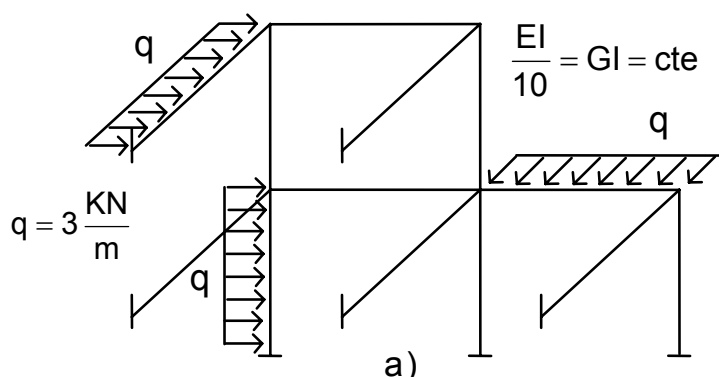

a)

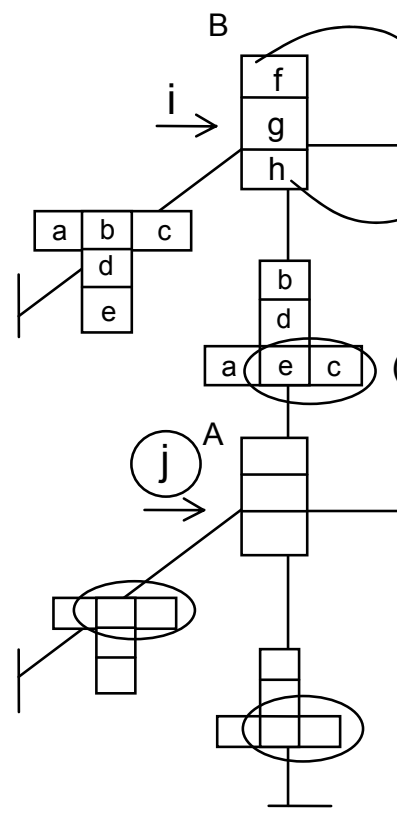

$\mathrm{C}$$$
\text { . }
$$

b)<smiles>C1CCC1</smiles>

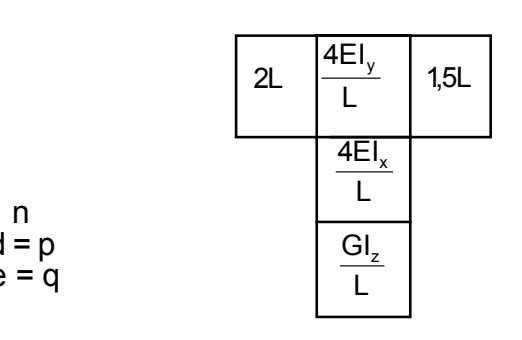

d)

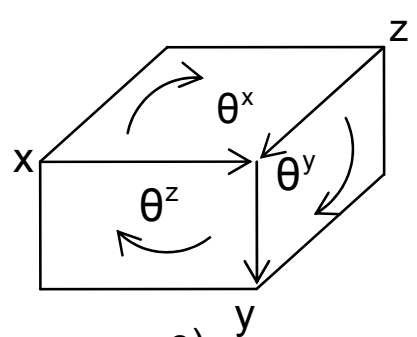

c)

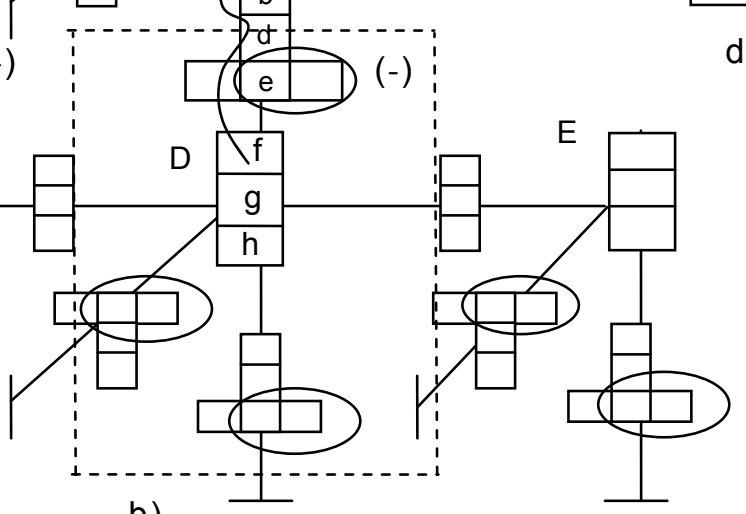

Fig. 4. Cálculo de momentos restrictivos en un pórtico: a) geometría, cargas y rigideces del modelo; b) esquema del pórtico, nomenclatura y reglas nemotécnicas utilizadas; c) criterios de signo positivo de acciones y de movimientos de los nudos; d) ejemplo de conjunto a 


\section{Obtención de las deformadas parciales}

Las acciones restrictivas de los estados primarios que se van a utilizar en el cálculo se expresan en función de las acciones activas correspondientes. Para ello es necesario conocer las deformadas parciales de estos estados primarios. Dichas deformadas se han calculado en función de acciones activas unitarias en la figura $4 \mathrm{~b}$ con ayuda de ciertas reglas nemotécnicas. Las deformadas producidas por los momentos activos unitarios se representan en las casillas $\mathrm{f}, \mathrm{g}$, $\mathrm{h}$ de cada nudo, y las producidas por las fuerzas unitarias en las casillas i, j. Por ejemplo, si la acción activa es un momento unitario que está orientado en el plano $X Z$ y aplicado en un nudo $n$, la deformada depende del giro $\theta_{n}^{\prime x}$ que vale (3).

$\theta_{n}^{\prime x}=\frac{M_{n}^{x}}{K_{n}^{x}}$

$\mathrm{K}_{\mathrm{n}}^{\mathrm{x}}$ es la suma de las rigideces a flexión en el plano $\mathrm{XZ}$ de todos los tramos que convergen en $\mathrm{n}$, es decir, la suma de los coeficientes b próximos a $n$ (Fig.4b). El resultado se dispone en la casilla homóloga de $n$, que en este caso es la casilla f. Cuando la acción activa es una fuerza unitaria aplicada en $n$, la deformada está determinada por el desplazamiento $\delta_{n}^{\prime}(4)$.

$$
\delta_{n}^{\prime}=\frac{F_{n}}{K_{n}}
$$

$\mathrm{K}_{\mathrm{n}}$ es la suma de los términos $\frac{12 \mathrm{El}}{\mathrm{L}^{3}}$ procedentes de los cortantes de las barras que giran por dicho desplazamiento. En la figura $4 b$, esta suma es la de los productos a.b.c y a.e.c de las barras unidas al tramo desplazable donde se encuentra $\mathrm{n}$. El resultado se dispone en la casilla situada sobre el vector cercano a $n$ que, en la figura $4 \mathrm{~b}$ es la casilla i o la $\mathrm{j}$.

Obtención de los momentos activos parciales $\mathrm{m}_{\mathrm{nM}}^{\mathrm{a}}, \mathrm{m}_{\mathrm{nB}}^{\mathrm{a}}$ y $\mathrm{m}_{\mathrm{nA}}^{\mathrm{a}}$

Para calcular estos momentos utilizando el procedimiento aproximado, es necesario determinar previamente las matrices iterativas correspondientes. En el ejemplo que se analiza, solamente se han obtenido las matrices relacionadas con los planos OXZ y OXY, ya que las cargas aplicadas no producen momentos en el plano restante. Como se comentó anteriormente, estas matrices establecen la relación que existe entre los momentos restrictivos $n_{n}$ y $q_{n}$ (Fig.4b) existentes en la estructura y los momentos activos $m_{n}^{a x}$ y $m_{n}^{a z}$ de los estados primarios que deben añadirse para eliminar $n_{n}$ y $q_{n}$. Los momentos $m_{n}^{a x}$ y $m_{n}^{a z}$ se obtienen mediante una regla nemotécnica que se ha aplicado a las barras BC y DC y cuyos valores se sitúan a ambos lados de las rigideces de dichas barras. Ordenándolos por nudos y planos se obtienen las expresiones (5) y (6) que se utilizarán posteriormente para determinar los momentos activos parciales.

$$
\begin{aligned}
& \left(\begin{array}{l}
\mathrm{n}_{\mathrm{A}} \\
\mathrm{n}_{\mathrm{B}} \\
\mathrm{n}_{\mathrm{C}} \\
\mathrm{n}_{\mathrm{D}} \\
\mathrm{n}_{\mathrm{E}}
\end{array}\right)=\left(\begin{array}{ccccc}
0 & 0,01 & 0 & 0,16 & 0 \\
0,01 & 0 & 0,24 & 0 & 0 \\
0 & 0,24 & 0 & 0 & 0 \\
0,24 & 0 & 0,01 & 0 & 0,24 \\
0 & 0 & 0 & 0,16 & 0
\end{array}\right) \times\left(\begin{array}{l}
\mathrm{m}_{\mathrm{A}} \\
\mathrm{m}_{\mathrm{B}} \\
\mathrm{m}_{\mathrm{C}} \\
\mathrm{m}_{\mathrm{D}} \\
\mathrm{m}_{\mathrm{E}}
\end{array}\right)^{\mathrm{ax}} \\
& \left(\begin{array}{l}
\mathrm{q}_{\mathrm{A}} \\
\mathrm{q}_{\mathrm{B}} \\
\mathrm{q}_{\mathrm{C}} \\
\mathrm{q}_{\mathrm{D}} \\
\mathrm{q}_{\mathrm{E}}
\end{array}\right)=\left(\begin{array}{ccccc}
0 & -0,24 & 0 & -0,12 & 0 \\
0,01 & 0 & -0,24 & 0 & 0 \\
0 & -0,24 & 0 & -0,12 & 0 \\
-0,16 & 0 & -0,24 & 0 & -0,24 \\
0 & 0 & 0 & -0,12 & 0
\end{array}\right) \times\left(\begin{array}{c}
\mathrm{m}_{\mathrm{A}} \\
\mathrm{m}_{\mathrm{B}} \\
\mathrm{m}_{\mathrm{C}} \\
\mathrm{m}_{\mathrm{D}} \\
\mathrm{m}_{\mathrm{E}}
\end{array}\right)^{\mathrm{az}}
\end{aligned}
$$


Los momentos $r_{n B}$ y $r_{n A}$ de los estados I y II homólogos a los de la figura 2 se obtienen en la figura $4 \mathrm{~b}$ a partir de las deformadas parciales utilizando otra regla nemotécnica. A modo de ejemplo, en la figura $4 \mathrm{~b}$ se explica la obtención de los momentos $r_{n A}$ : se multiplica j por las casillas marcadas con un ovalo pertenecientes a las barras conectadas al dintel desplazable ADE. Todos los productos son positivos salvo los que afectan a las casillas que están por encima de j según la figura, y los momentos $r_{n A}$ de cada nudo se representan en las proximidades de dicho nudo. Por ejemplo, los momentos $r_{D A}$ se muestran recogidos en el perímetro discontinuo del entorno de $D$. La orientación en el espacio de cada momento depende de la orientación de la barra de la cual proceda. De esta manera, los que provienen de las barras paralelas al eje OZ están en planos paralelos al OXZ, y los que proceden de barras paralelas al eje OY son paralelos al OXY. En las expresiones (7) y (8) se han recogido los valores de todos los momentos calculados.

$$
\begin{aligned}
& \left(\begin{array}{l}
\mathrm{m}_{\mathrm{A}} \\
\mathrm{m}_{\mathrm{B}} \\
\mathrm{m}_{\mathrm{C}} \\
\mathrm{m}_{\mathrm{D}} \\
\mathrm{m}_{\mathrm{E}}
\end{array}\right)^{a x}=\left(\begin{array}{ll}
\mathrm{r}_{\mathrm{AB}} & \mathrm{r}_{\mathrm{AA}} \\
\mathrm{r}_{\mathrm{BB}} & \mathrm{r}_{\mathrm{BA}} \\
\mathrm{r}_{\mathrm{CB}} & \mathrm{r}_{\mathrm{CA}} \\
\mathrm{r}_{\mathrm{DB}} & \mathrm{r}_{\mathrm{DA}} \\
\mathrm{r}_{\mathrm{EB}} & \mathrm{r}_{\mathrm{EA}}
\end{array}\right)^{x}=\left(\begin{array}{cc}
0 & 0,24 \\
0,49 & 0 \\
0,49 & 0 \\
0 & 0,24 \\
0 & 0,24
\end{array}\right)^{x} \\
& \left(\begin{array}{l}
\mathrm{m}_{\mathrm{A}} \\
\mathrm{m}_{\mathrm{B}} \\
\mathrm{m}_{\mathrm{C}} \\
\mathrm{m}_{\mathrm{D}} \\
\mathrm{m}_{\mathrm{E}}
\end{array}\right)^{\mathrm{az}}=\left(\begin{array}{ll}
\mathrm{r}_{\mathrm{AB}} & \mathrm{r}_{\mathrm{AA}} \\
\mathrm{r}_{\mathrm{BB}} & \mathrm{r}_{\mathrm{BA}} \\
\mathrm{r}_{\mathrm{CB}} & \mathrm{r}_{\mathrm{CA}} \\
r_{\mathrm{DB}} & \mathrm{r}_{\mathrm{DA}} \\
r_{\mathrm{EB}} & \mathrm{r}_{\mathrm{EA}}
\end{array}\right)^{z}=\left(\begin{array}{cc}
0,49 & 0 \\
0,49 & -0,24 \\
0,49 & -0,24 \\
0,49 & 0 \\
0 & 0,24
\end{array}\right)^{z}
\end{aligned}
$$

Con (5), (6), (7) y (8) y los momentos $m_{n M}^{a x}$ y $m_{n M}^{a z}$ obtenidos a partir de las cargas exteriores se calculan en (9) y (10) los momentos activos parciales realizando tres iteraciones.

$$
\begin{aligned}
& \left(\begin{array}{ccccc}
0 & 0,01 & 0 & 0,16 & 0 \\
0,01 & 0 & 0,24 & 0 & 0 \\
0 & 0,24 & 0 & 0,008 & 0 \\
0,24 & 0 & 0,01 & 0 & 0,24 \\
0 & 0 & 0 & 0,16 & 0
\end{array}\right) \cdot\left(\begin{array}{ccc}
0 & 0 & 0,24 \\
4 & 0,49 & 0 \\
0 & 0,49 & 0 \\
4 & 0 & 0,24 \\
4 & 0 & 0,24
\end{array}\right)=\left(\begin{array}{ccc}
0,68 & 0 & 0,03 \\
0 & 0,11 & 0 \\
0,99 & 0,11 & 0 \\
0,96 & 0 & 0,11 \\
0,64 & 0 & 0,03
\end{array}\right)+\ldots+\left(\begin{array}{ccc}
0,05 & 0 & 0 \\
0 & 0 & 0 \\
0 & 0 & 0 \\
0 & 0 & 0 \\
0,05 & 0 & 0
\end{array}\right)=\left(\begin{array}{cccc}
-0,88 & 0 & 0,21 \\
-4,25 & 0,39 & 0 \\
1,07 & 0,39 & 0 \\
5,36 & 0 & 0,13 \\
-4,84 & 0 & 0,21
\end{array}\right)^{x} \\
& \left(\begin{array}{ccccc}
0 & -0,24 & 0 & -0,12 & 0 \\
0,01 & 0 & -0,24 & 0 & 0 \\
0 & -0,24 & 0 & -0,12 & 0 \\
-0,16 & 0 & -0,24 & 0 & -0,24 \\
0 & 0 & 0 & -0,12 & 0
\end{array}\right) \cdot\left(\begin{array}{ccc}
-4 & 0,49 & 0 \\
0 & 0,49 & -0,24 \\
0 & 0,49 & -0,24 \\
0 & 0,49 & 0 \\
0 & 0 & 0,24
\end{array}\right)=\left(\begin{array}{ccc}
0 & -0,17 & 0,05 \\
0,64 & -0,19 & 0,05 \\
0 & -0,17 & 0,05 \\
0,64 & -0,19 & 0 \\
0 & -0,05 & 0
\end{array}\right)+\ldots+\left(\begin{array}{cccc}
0 & -0,02 & 0 \\
0,06 & -0,02 & 0 \\
0,07 & -0,02 & 0 \\
0,11 & -0,03 & 0 \\
0 & -0,01 & 0
\end{array}\right)=\left(\begin{array}{ccc}
-4,23 & 0,35 & 0,05 \\
0,73 & 0,33 & -0,19 \\
-0,23 & 0,35 & -0,18 \\
0,75 & 0,34 & -0,01 \\
-0,07 & -0,04 & 0,24
\end{array}\right)^{2} \mathrm{mKN}
\end{aligned}
$$

A diferencia del método de Cross y como sucede con los métodos de Kani y de Takabeya, en este procedimiento se calcula únicamente un factor en cada nudo por cada iteración. Esto agiliza la realización de las iteraciones. Desde este punto de vista, el método se hace más útil a medida que se analizan figuras con más cantidad de nudos o bien cuando existen nudos en los que convergen muchas barras. Como complemento a este cálculo se han determinado los valores exactos de estos momentos activos utilizando unas tablas similares a las Tablas 1 y 2 , cuyos resultados se muestran en (11) y (12).

$$
\left(\begin{array}{ccc}
m_{A M} & m_{A B} & m_{A A} \\
m_{B M} & m_{B B} & m_{B A} \\
m_{C M} & m_{C B} & m_{C A} \\
m_{D M} & m_{D B} & m_{D A} \\
m_{E M} & m_{E B} & m_{E A}
\end{array}\right)^{a x}=\left(\begin{array}{ccc}
-0,90 & 0 & 0,21 \\
-4,25 & 0,39 & 0 \\
1,02 & 0,39 & 0 \\
5,39 & 0 & 0,13 \\
-4,86 & 0 & 0,21
\end{array}\right) \mathrm{mKN}
$$


$\left(\begin{array}{ccc}\mathrm{m}_{A M} & \mathrm{~m}_{A B} & \mathrm{~m}_{A A} \\ \mathrm{~m}_{\mathrm{BM}} & \mathrm{m}_{\mathrm{BB}} & \mathrm{m}_{\mathrm{BA}} \\ \mathrm{m}_{\mathrm{CM}} & \mathrm{m}_{\mathrm{CB}} & \mathrm{m}_{\mathrm{CA}} \\ \mathrm{m}_{\mathrm{DM}} & \mathrm{m}_{\mathrm{DB}} & \mathrm{m}_{\mathrm{DA}} \\ \mathrm{m}_{\mathrm{EM}} & \mathrm{m}_{\mathrm{EB}} & \mathrm{m}_{\mathrm{EA}}\end{array}\right)^{\mathrm{z}}=\left(\begin{array}{ccc}-4,27 & 0,36 & 0,05 \\ 0,74 & 0,34 & -0,2 \\ -0,27 & 0,36 & -0,18 \\ 0,77 & 0,35 & -0,02 \\ -0,09 & -0,04 & 0,24\end{array}\right)^{z} \mathrm{mKN}$

Se observa que los resultados aproximados no divergen por término medio más de un 2,5\% respecto de los exactos, salvo en los casos de $m_{C M}^{z}, m_{E M}^{z} y m_{D A}^{z}$, en los que el error alcanza un 14,8 , un 22 y un $50 \%$, respectivamente. Estas desviaciones no parecen relevantes ya que los valores que se están comparando son demasiado pequeños. Sin embargo, si hubiera que analizar el comportamiento de la figura frente a diferentes alternancias de carga, el procedimiento exacto podría ser igualmente adecuado, ya que bastaría con utilizar una sola vez las tablas para obtener los momentos activos exactos en función de unos momentos genéricos exteriores.

\section{Obtención de las fuerzas activas $\mathrm{f}_{\mathrm{A}}^{\mathrm{a}}$ y $\mathrm{f}_{\mathrm{B}}^{\mathrm{a}}$}

Los procedimientos aproximado y exacto calculan las fuerzas activas partiendo de las fuerzas $B B$, $A B$ y $B A, A A$ del pórtico que son homólogas a las de las figuras $2 f$ y $2 h$, y por tanto están formadas por las fuerzas restrictivas de los estados primarios I y II y por las debidas a la eliminación de los momentos restrictivos en dichos estados. $B B, A B$ y $B A$, $A A$ se determinan en la figura 5 utilizando dos reglas nemotécnicas. Una de ellas calcula las fuerzas restrictivas de los estados primarios I y II, y se ha aplicado en la figura 5a para determinar las fuerzas restrictivas del estado II. Sabiendo que la fuerza en A es nula, la fuerza en B se obtiene multiplicando el coeficiente j por la suma de los productos a.e.c de todos los grupos a de las barras comprendidas entre los dos grupos de barras desplazables. Estos resultados junto con los obtenidos para el estado I, se recogen en la primera matriz de (13). Por otra parte, la segunda regla nemotécnica calcula las fuerzas restrictivas producidas al aparecer los momentos $m_{n M}^{a}, m_{n A}^{a}$ y $\mathrm{m}_{\mathrm{nB}}^{\mathrm{a}}$. Dicha regla se ha empleado en la figura $5 \mathrm{~b}$ para conocer las fuerzas en $A$ y $B$ debidas a unos momentos activos unitarios en $\mathrm{B}$ y $\mathrm{C}$. En el pórtico únicamente influyen los momentos en OXZ de las barras paralelas al OZ y los momentos OXY de las barras paralelas al OY. Multiplicando las casillas a.b.c por las casillas $f$ de $B$ y $C$ se obtiene la influencia de $m_{B B}^{a x}$ y $m_{C C}^{a x}$ en la fuerza restrictiva de B. Por otro lado, multiplicando las casillas a.e.c por las casillas $h$ de B y $C$ se obtienen dos fuerzas iguales y de signo contrario que representan la incidencia de $m_{B B}^{a z}$ y $\mathrm{m}_{\mathrm{CC}}^{\mathrm{az}}$ en $\mathrm{A}$ y $\mathrm{B}$. Si B y $\mathrm{C}$ hubieran pertenecido a un dintel desplazable situado entre otros dos desplazables, como le sucede al nudo $\mathrm{n}$ de la figura $5 \mathrm{c}$, los signos de las fuerzas restrictivas hubieran sido como aparecen en dicha figura. Con estas reglas se han obtenido las fuerzas restrictivas de los estados $I I$ y I y las producidas por $m_{n M}^{a}$, que se recogen en las segundas matrices de (13) y (14), respectivamente. En (13) se ha calculado la matriz iterativa y en (14) se han determinado las fuerzas activas similares a las de la figura $2 \mathrm{~d}$. La matriz iterativa establece la relación entre las fuerzas restrictivas existentes y las fuerzas activas de los estados que se añaden en cada iteración.

$$
\begin{aligned}
& \left(\begin{array}{cc}
0 & 0,24 \\
0,49 & 0
\end{array}\right)+\left(\begin{array}{cc}
0,34 & -0,06 \\
-0,13 & 0,18
\end{array}\right)=\left(\begin{array}{cc}
0,34 & 0,18 \\
0,36 & 0,18
\end{array}\right)=\left(\begin{array}{cc}
B B & B A \\
A B & A A
\end{array}\right) \\
& \left(\begin{array}{l}
P_{B} \\
P_{A}
\end{array}\right)+\left(\begin{array}{l}
B M \\
A M
\end{array}\right)=\left(\begin{array}{l}
6 \\
6
\end{array}\right)+\left(\begin{array}{c}
-0,92 \\
-0,49
\end{array}\right)=\left(\begin{array}{c}
5,07 \\
5,5
\end{array}\right)=\left(\begin{array}{l}
F_{B} \\
F_{A}
\end{array}\right)
\end{aligned}
$$

Con (13) y (14), en (15) se calculan los valores aproximados de las fuerzas activas realizando siete iteraciones. 
$\left(\begin{array}{ll}0,34 & 0,18 \\ 0,36 & 0,18\end{array}\right) \cdot\left(\begin{array}{c}5,07 \\ 5,5\end{array}\right)=\left(\begin{array}{l}2,71 \\ 2,81\end{array}\right)+\left(\begin{array}{c}1,43 \\ 1,48\end{array}\right)+\ldots .+\left(\begin{array}{c}0,05 \\ 0,05\end{array}\right)=\left(\begin{array}{c}10,71 \\ 11,35\end{array}\right) \mathrm{KN}$

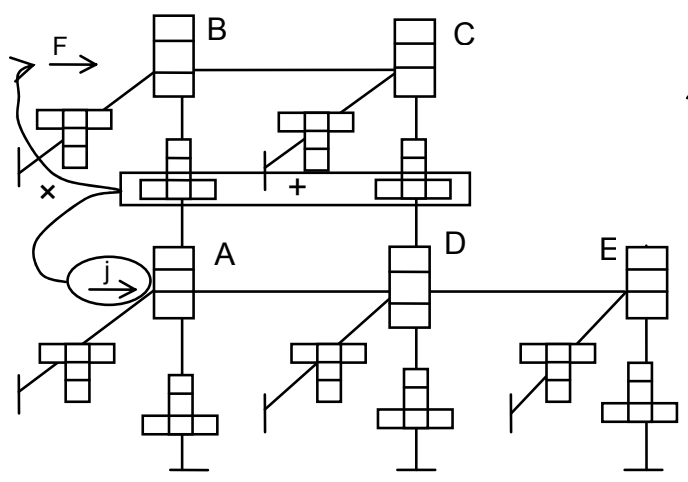

a)

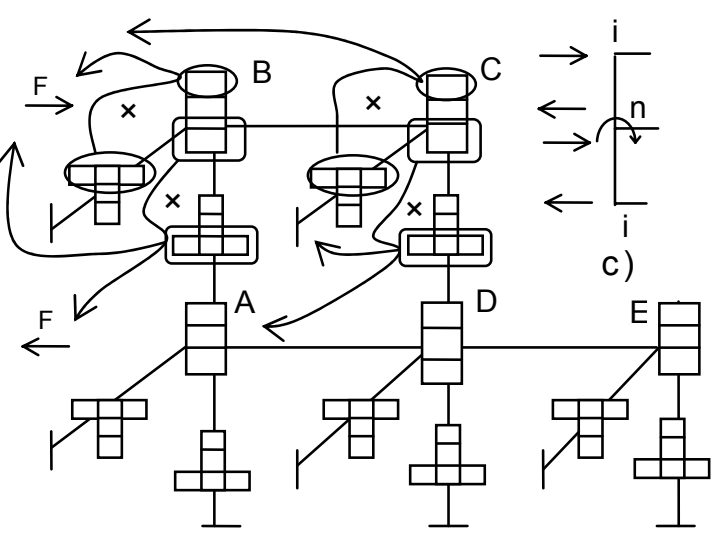

b)

Fig. 5. Cálculo de fuerzas restrictivas $F$ en un pórtico mediante reglas nemotécnicas: a) por un estado primario con fuerza activa unitaria en $A, b$ ) por momentos unitarios en $B$ y $C$; c) sentido de las fuerzas restrictivas en $\mathrm{i}, \mathrm{j}$, $\mathrm{n}$ por un momento en $\mathrm{n}$

Comparando estos resultados con los valores exactos mostrados en (16), que están obtenidos a partir de los datos de (13) y de las Tablas 1 y 2 , se observa que los resultados aproximados de las fuerzas tampoco se desvían esencialmente respecto de los exactos. Con (16) y con las expresiones de los momentos activos de la figura $2 \mathrm{i}$, se obtienen los momentos activos finales. A partir de ellos, con (3), (4) y (16), queda determinada la deformada y los esfuerzos de los tramos.

$$
\left(\begin{array}{c}
f_{B}^{a} \\
f_{A}^{a}
\end{array}\right)=\left(\begin{array}{c}
10,80 \\
11,45
\end{array}\right) K N
$$

\section{CONCLUSIONES}

El presente trabajo demuestra que, descomponiendo las acciones exteriores actuantes en sus estados de carga primarios, se puede realizar un análisis estructural exacto en teoría de primer orden de pórticos ordinarios de edificación sin resolver sistemas de ecuaciones.

Con el procedimiento aproximado se consiguen unos resultados suficientemente precisos realizando un número reducido de iteraciones, siendo el error cometido estimable en todo momento.

\section{REFERENCIAS}

Aristizabal-Ochoa, J.D, Estabilidad y análisis de segundo orden de estructuras de vigas y columnas de Timoshenko con conexiones semirrígidas: método pendiente-deflexión, Dyna rev.fac.nac.minas: 76(159), 7-21(2009).

Calavera, J., Proyecto y cálculo de estructuras de hormigón, vol I Ed. Intemac, pp 229-231, Madrid, España (1999).

Fraile de Lerma, A., L.K.H. Hermanns y E. Alarcón, El cálculo de estructuras en la obra de Carlos Fernández Casado, Informes de la Construcción: 60(509), 45-56 (2008).

Giordano, A y otros tres autores, A simple formula for predicting the horizontal capacity of masonry portal frames, Engineering Structures: 29(9), 2109-2123 (2007). 
Grinter, L.E., Métodos clásicos de estructuras indeterminadas, Estructuras modernas de acero, Ed. Diana, pp 419-451, México, D.F., México (1969).

Günay, M.S y H. Sucuoglu, An improvement to linear-elastic procedures for seismic performance assessment, Earthquake Engineering \& Structural Dynamics: 39(8), 907-931 (2010).

Lacort, A.G., Análisis manual de emparrillados mediante cargas descompuestas, Libro de comunicaciones del IV Congreso Internacional de Estructuras ACHE, 303, Valencia, España, 24 a 27 de Noviembre (2008).

Portero, J.C. y otros seis autores, Números gordos en el proyecto de estructuras, Ed. Cinter divulgación científica, pp 5-6, Madrid, España (2004).

Takabeya, F., Estructuras de varios pisos sometidas a cargas verticales y horizontales, Estructuras de varios pisos: cálculo y tablas de momentos: métodos de Cross, Kani y Takabeya, Ed. CECSA, pp 3-73, Barcelona, España (1968).

Zubydan, A.H, A simplified model for inelastic second order analysis of planar frames, Engineering Structures 32(10), 3258-3268 (2010). 
